\title{
Philosophiques
}

\section{Descartes face à Leibniz sur la question de la substance}

\section{Josiane Boulad Ayoub}

Volume 11, numéro 2, octobre 1984

Égalité, justice et différence

URI : https://id.erudit.org/iderudit/203256ar

DOI : https://doi.org/10.7202/203256ar

Aller au sommaire du numéro

Éditeur(s)

Société de philosophie du Québec

ISSN

0316-2923 (imprimé)

1492-1391 (numérique)

Découvrir la revue

Citer cet article

Boulad Ayoub, J. (1984). Descartes face à Leibniz sur la question de la substance. Philosophiques, 11(2), 225-249. https://doi.org/10.7202/203256ar

\section{Résumé de l'article}

Il s'agit de montrer l'équivocité de la notion de substance quand on la prend à la fois chez Descartes et chez Leibniz, et de comparer à propos de ce problème les positions des deux philosophes. La question est abordée sous l'angle du rapport entre les modifications de rôle et de sens de la notion de substance telle qu'elles apparaissent chez les deux représentants des tendances constitutives de l'epistême classique. La confrontation sert à préciser, d'une part, les déterminations ontologiques du maintien de cette notion dans les problématiques respectives de Descartes et de Leibniz; d'autre part, elle vise à alimenter d'une pièce supplémentaire le débat sur la différence entre les enjeux du rationalisme cartésien et celui leibnizien. 


\title{
ARTICLES
}

\section{DESCARTES FACE À LEIBNIZ SUR LA QUESTION DE LA SUBSTANCE}

\author{
par Josiane Boulad Ayoub
}

\begin{abstract}
RÉSUMÉ. Il s'agit de montrer l'équivocité de la notion de substance quand on la prend à la fois chez Descartes et chez Leibniz, et de comparer à propos de ce problème les positions des deux philosophes. La question est abordée sous l'angle du rapport entre les modifications de rôle et de sens de la notion de substance telle qu'elles apparaissent chez les deux représentants des tendances constitutives de l'epistême classique. La confrontation sert à préciser, d'une part, les déterminations ontologiques du maintien de cette notion dans les problématiques respectives de Descartes et de Leibniz ; d'autre part, elle vise à alimenter d'une pièce supplémentaire le débat sur la différence entre les enjeux du rationalisme cartésien et celui leibnizien.
\end{abstract}

\begin{abstract}
We will first examine the manner in which Descartes and Leibniz each defines the notion of substance ; then the meaning that each of these authors attributes to this notion ; finally, we shall analyze the relations between substances in their respective systems. This comparison should serve to throw light on the ontological determinations of this notion as it appears in the respective systems of Descartes and Leibniz as well as to differenciate between the rationalism of each to them.
\end{abstract}

Le problème du Même et de l'Autre se pose aussi, d'une certaine façon, pour les historiens de la philosophie! La multiplicité des investissements opérée, dans l'histoire, par le discours philosophique à partir d'un capital commun et identique de notions, concepts ou catégories qu'on aurait pu croire immuables, oblige l'analyse historique comparée à des tâches délicates : identifier les changements d'acception à travers les problématiques des auteurs considérés, mettre au jour leurs 
déterminations ontologico-épistémiques, voire leurs enjeux idéologico-politiques. Ce sont là quelques exemples des difficultés auxquelles se heurte ce type d'analyse et que viennent redoubler celles causées par l'interprétation elle-même : les postulats méthodologiques qu'elle adopte, le point de vue, diachronique ou synchronique auquel elle se place.

Cette question générale de la variété des exploitations rencontrée par la même notion à travers les diverses philosophies, sans parler de son inévitable polysémie ou encore de ses acceptions changeantes à l'intérieur, cette fois, d'une même doctrine, revêt une acuité particulière lorsqu'il s'agit de la notion de substance : non seulement parce que cette notion tient une place assez éminente dans le patrimoine commun, que j'évoquais tout à l'heure, pour révéler de manière significative ce problème du changement dans la permanence, non seulement parce qu'elle enregistre, dans les principes mêmes de son procès, un nombre assez considérable de développements pour marquer les lieux du passage du mode de pensée substantialiste au mode de pensée relationnelle et, au-delà, systémique, caractéristique de notre post-modernité, mais surtout parce qu'elle constitue un de ces points nodaux autour desquels s'organise un système philosophique et au sujet desquels se font les lignes les plus symptomatiques de partage.

Je voudrais, précisément, montrer ici l'équivocité de la notion de substance dans la philosophie moderne quand on la prend à la fois chez Descartes et chez Leibniz, et comparer à propos de ce problème les positions des deux philosophes. (Certes, la complexité de la question générale en jeu aurait dû entraîner des considérations plus longues et plus complètes que celles qui suivront dans le corps de l'étude ou que la longueur des présents propylées - auxquels m'obligent les remarques judicieuses et amicales du comité de lecture pour préciser mes intentions aurait pu faire espérer.) Qu'on m'entende bien : a) je limite ma contribution à l'étude d'un cas particulier à l'intérieur du modèle philosophique classique dont Descartes et Leibniz comptent parmi les figures les plus représentatives de ses variantes structurelles ; de plus ces deux philosophes partagent, en dépit du fait qu'un demi-siècle les sépare, un socle épistémique commun, ainsi que l'appellerait Foucault : dès lors on peut confronter, à partir de 
ce point de vue synchronique auquel je me place dans le cadre de cette analyse comparative, leurs thèses respectives sur la notion de substance ; b) je n'ai pas voulu reconstituer tous les éléments de la doctrine de la substance chez l'un et chez l'autre philosophe, ni non plus exposer leurs sources en détail (langage mathématique et dynamique du Discours de Métaphysique par exemple) ni, a fortiori, rendre compte de la « littérature secondaire » sur le sujet puisqu'il ne s'agissait pas, pour moi, de faire une étude des interprétations des commentateurs, si importants fussentils, telles celles des Gueroult, Russell, Belaval ou de mon maître Rodis-Lewis, mais bien de comparer brièvement, sans surcharge d'érudition, sur un point particulier, Descartes et Leibniz. Suivre exhaustivement le détail des changements qui s'établissent d'un type d'argumentation à l'autre dans les ouvrages des auteurs considérés - et pour Leibniz il se serait agi de suivre plusieurs séries d'arguments - aurait exigé d'infliger au lecteur un texte à la longueur démesurée par rapport aux finalités d'un article de revue, en général, et aux objectifs particuliers - et modestes - de celui-ci : être autant que possible concise, claire, en un mot, éviter d'être " pédestre " comme disent nos amis anglais ! Attirer l'attention de ceux qui lisent Philosophiques sur un point de l'histoire de la philosophie, la notion de substance, qui retrouve aujourd'hui un regain d'intérêt avec le débat sur les concepts de l'ontologie contemporaine. Aborder cette question sous l'angle du rapport entre les modifications de rôle et de sens de la notion de substance telles qu'elles apparaissent chez deux philosophes modernes. Je n'ai pas cherché à montrer la nouveauté des conceptions de la substance chez ces philosophes par rapport à la scolastique : par exemple, expliquer comment et pourquoi Leibniz ne retient des définitions de la tradition aristotélicienne de la substance comme ultimum subsistens ou ultimum perdurabile qui se retrouvera, par ailleurs, discutée par Kant, soit dit en passant - que la troisième définition, comme vis activa. En revanche, ce que j'ai cherché surtout à faire c'est une comparaison - non une juxtaposition des doctrines - à partir du centre de l'intuition première dont s'inspire tout le développement ultérieur de la pensée et des conceptions de l'un et de l'autre philosophe. 
Il demeure assez tentant d'établir une comparaison entre Descartes et Leibniz, même avec les limites que j'ai signalées ci-dessus, à propos du traitement de la notion de substance par chacun d'eux. Cette comparaison a son intérêt : une notion non seulement scolastique mais antique est maintenue dans la philosophie moderne à sa genèse alors qu'elle semblerait dépassée en cette période de changement de paradigmes ; à partir de ce fait, la comparaison servira à préciser, d'une part, les déterminations ontologico-épistémisques de ce maintien dans les problématiques respectives de Descartes et de Leibniz ; en quel sens la notion de substance est réinterprétée par ces deux philosophes et comment elle est exploitée par eux en regard des tendances constitutives de l'epistème classique ainsi que de la théorie naissante du sujet; d'autre part la comparaison vise à alimenter d'une pièce supplémentaire le débat sur la différence entre les enjeux du rationalisme cartésien et celui leibnizien : la distinction que Descartes fait entre res cogitans et res extensa, par exemple, ne signifiant pas seulement que la res cogitans est une substance - ceci Leibniz l'acceptera - mais surtout que la res extensa constitue aussi une substance. C'est à quoi s'oppose Leibniz, après son séjour à Paris et ses travaux en mathématique et en physique : si la substance est définie par lui, ainsi qu'on le verra, comme activité et spontanéité, alors la res extensa ne peut être substance, alors le mécanisme cartésien qui soutient cette conception ne vaut plus comme explication scientifique; pour Leibniz, la substance est un être vivant, et sa dynamique s'appuiera sur son refus initial d'admettre les substances inorganiques.

On peut commencer à noter le contraste entre la notion de substance chez Descartes et chez Leibniz en suivant son chemin respectif d'apparition chez chacun de ces deux philosophes. Dans la Seconde Méditation, préparée par ce qui a été avancé dans les Regulae et dans le Discours, Descartes ne tient guère à reprendre une telle notion appartenant au bagage de la scolastique; il marque sa répugnance à traiter scolastiquement de la question ; il refuse de prendre au sérieux la définition de l'homme, animal raisonnable, et à suivre son texte, on le voit parler d'une res cogitans, d'une res extensa, mais pas de la substance au sens sco- 
lastique du terme ${ }^{1}$. Ce ne sera que plus tard, lorsque Descartes se croira obligé de donner un manuel pour remplacer, par la "Vraie philosophie ", les manuels de scolastique, qu'il reprendra - reluctans - la notion de substance, dans les Principes (1644).

La situation est bien différente, en revanche, pour Leibniz. Celui-ci témoigne un grand accueil aux notions scolastiques, surtout à la notion de substance qu'il place d'assez bonne heure au centre de ses préoccupations; peut-être dès son voyage à Paris, vers l'âge de 25 ans, lorsqu'il prend contact avec la mathématique moderne. Leibniz n'entend pas, comme Descartes, renier l'héritage greco-scolastique. Les vieux problèmes de la tradition métaphysique seront réexaminés dans cette variété et cette multiplicité de perspectives - pourtant unifiées, car tout " conspire " comme il le dit, dans sa philosophie - qui sont la marque propre de son génie encyclopédique, et renouvelés au moyen de ses connaissances considérables, théologiques, politiques, scientifiques, logiques, philosophiques ... Ainsi la question de la substance, en particulier, sera exploitée sous un triple et même quadruple aspect : théologique, physique, logique et métaphysique ${ }^{2}$. Et contre Locke qui dénonce l'obscurité et le peu d'usage dans la philosophie moderne de l'idée de substance ${ }^{3}$, Leibniz, dans les Nouveaux Essais (1700), réaffirme sa position : «J'avoue que je suis d'un autre sentiment, et je crois que la

1. Je reviendrai sur les rapports entre cogito et substance dans ma section sur la définition de la substance par Descartes : ici je veux seulement faire remarquer combien il faut attendre pour tenir une conception pleine, techniquement parlant, de la substance chez Descartes.

2. On le voit, Leibniz accueille d'emblée, et très tôt, au contraire de Descartes, la notion de substance. Je signale ici, à l'attention du lecteur, les textes principaux où se trouvent consignées les thèses leibniziennes relatives à la notion de substance. Dans le corps de mon article, je me concentre surtout sur celles du Discours de Métaphysique. Je donne, à titre indicatif, cette liste de textes, dans l'ordre chronologique mais sans marquer ni analyser les distinctions entre les thèses : comme le fait remarquer justement mon anonyme lecteur à qui je dois aussi la liste qui suit, "nous manquons pour Leibniz, en particulier, d'une bonne analyse des systèmes successifs d'arguments relatifs à la notion de substance " ce serait peut-être l'occasion d'un bon sujet de programme de recherche. Les thèses initiales, le noyau de la doctrine leibnizienne, sont celles du De principio individui, de la correspondance avec Thomasius et avec Hobbes ; Leibniz établit ensuite les thèses impliquées par la mise au point de la dynamique, puis viennent les thèses centrales du Discours de Métaphysique ainsi que des textes qui en découlent, puis les arguments critiques contenus dans les Nouveaux Essais contre Locke, enfin les modifications apportées dans la version monadologique du système.

3. L'opinion de Locke sur la notion de substance est résumée par Leibniz dans les Nouveaux Essais sur l'Entendement, II, 12, 6. 
considération de la substance est un point des plus importants et des plus féconds de la Philosophie $"{ }^{4}$.

J'examinerai, tout d'abord, et c'est ma plus longue section, comment Descartes et Leibniz posent et définissent la notion de substance ; puis, ce que recouvre pour chacun d'eux cette notion, son contenu ; j'envisagerai, enfin, la question des rapports des substances entre elles, élément différentiel assez important dans les théories de tous ceux qu'on a appelé (pour marquer leur dette envers la matrice cartésienne) les grands cartésiens: Malebranche, Spinoza ou Leibniz.

\section{1- POSITIONS OU DÉFINITION DE LA SUBSTANCE.}

1.1 La notion de substance, chez Descartes, - ou du moins ce qui la remplace dans son esprit avant qu'il ne l'utilise explicitement - dans les Méditations, par exemple, concerne particulièrement la substance pensante. On se rappellera la conduite de la recherche à ce sujet, au courant de la Seconde Méditation ${ }^{5}$, lorsque, une fois affirmé le « je pense, je suis», Descartes se demande : mais que suis-je ? une chose qui pense ; mais qu'estce qu'une chose qui pense ? etc . . . Il n'aborde la notion d'une autre substance, la substance étendue, ou selon le langage des Méditations, la res extensa, qu'après et presque en vertu de la position de la chose pensante. Au niveau de la Seconde Méditation, la substance - la chose - étendue s'oppose à la substance pensante, mais aussi elle la suit. L'exemple du morceau de cire, et tout le développement qui lui est consacré, sert ce propos de Descartes : le problème de la substance étendue se pose en fonction du problème de la chose pensante. C'est pourquoi, en un certain sens, on peut dire que l'approche cartésienne du problème de la substance est une approche psychologique et humaniste ; mieux, ontologico-subjectiviste, pour éviter toute ambiguïté sur le terme psychologique.

Pour Leibniz, par contre, l'approche est tout d'abord logique. Le Discours de Métaphysique (1686 ?), écrit de la jeune maturité de Leibniz, et fondamental eu égard à sa philosophie,

4. In Nouveaux Essais sur l'Entendement, II, 13, 19.

5. Descartes, A.T. IX, 21, in Oeuvres de Descartes, publiées par Adam et Tannery, L. Cerf., 1897-1913, en 13 volumes. 
le montre bien. Le paragraphe VIII qui définit la notion de substance fait état de propositions, de prédicats et de sujets ${ }^{6}$. On ne peut parler de priorité, au sens strict, d'une substance sur une autre. Au paragraphe $\mathrm{XII}^{7}$, Leibniz montre même qu'on ne peut soutenir, comme Descartes l'a fait, que l'étendue soit une substance : elle n'a pas assez de réalité pour tenir en ellemême, pour exister, se perpétuer, avoir de l'unité, ce qui serait logiquement exigé par la définition de la substance, donnée au paragraphe VIII.

1.2 Si l'on envisage maintenant comment la substance est définie, conçue et expliquée chez l'un et chez l'autre philosophe, on verra le même contraste se développer et même devenir plus aigu.

La théorie de la substance n'est abordée qu'indirectement et qu'implicitement dans les Méditations, et j'ai dit, tout à l'heure, pourquoi ce n'est que dans les Principes que Descartes ne peut plus éviter de parler de la substance. Il n'en reste pas moins, qu'avant les Principes, Descartes est en possession du noyau, ou du moins, du point de départ essentiel d'une théorie concernant la substance, bien que toutes les explications telles que nous les donnent les Méditations, et aussi, en un certain sens, les Réponses aux Objections (particulièrement la Réponse à la $3^{e}$ objection formulée par Hobbes) et peut-être finalement toute la philosophie cartésienne, ne peuvent concerner que la « res cogitans", et même plus étroitement encore le moi.

1.3 J'ai rappelé, tout à l'heure, qu'à la question que Descartes se pose, après avoir formulé sa proposition célèbre, « je pénse, je suis », mais que suis-je ? il refusait de répondre : « je suis

6. Leibniz, paragraphe VIII in Discours de Métaphysique, ed. H. Lestienne, Vrin, 1970, $6^{\mathrm{e}}$ édition, pp. 35-36 :

. . . « Il est bien vrai que lorsque plusieurs prédicats s'attribuent à un même sujet, et que ce sujet ne s'attribue à aucun autre, on l'appelle substance individuelle . . Or il est constant que toute prédication véritable a quelque fondement dans la nature des choses, et lorsqu'une proposition n'est pas identique, c'est-à-dire lorsque le prédicat n'est pas compris expressément dans le sujet il faut qu'il y soit compris virtueilement, et c'est ce que les Philosophes appellent in-esse en disant que le prédicat est dans le sujet . . . Cela estant, nous pouvons dire que la nature d'une substance individuelle ou d'un estre complet est d'avoir une notion si accomplie qu'elle soit suffisante à comprendre et à en faire déduire tous les prédicats du sujet à qui cette notion est attribuée ».

7. Leibniz, op. cit. ; titre du paragraphe XII : que les notions qui consistent dans l'étendue enferment quelque chose d'imaginaire et ne sçauroient constituer la substance des corps. 
un homme donc un animal raisonnable » car cette dernière proposition entraînait un processus infini d'explications verbales ne débouchant nulle part. Descartes poursuit alors la question : que suis-je, moi qui pense que j'existe ? La réponse immédiate qui est : " je suis en tant que je pense " suscite l'objection suivante que se pose Descartes : mais ne suis-je seulement qu'en tant que je pense ? c'est-à-dire, est-ce que ma réalité ne se maintient qu'au moment où je pense ? N'aurais-je, par conséquent, qu'une existence à la fois empirique et atomique ? Non. Quand je dis que « j'existe en tant que je pense ", je veux dire par là que je suis une chose qui pense, c'est-à-dire, ajoute-t-il immédiatement, une substance pensante. Quelle valeur peut avoir, dans le contexte cartésien, une pareille affirmation ? Cela signifie qu'une fois libéré du doute et par le doute, je suis libéré aussi par la pensée et affirmé dans la pensée, ou plus clairement encore : je puis douter de tout sauf du fait que je suis une chose qui pense. Je suis assuré, de plus, que dans la mesure où je m'affirme comme une substance qui pense, je sais que je ne suis pas un corps ; et cette distinction est fondamentale : à l'affirmation du moi comme substance qui pense, elle ajoute que, même si, en ce moment, je ne suis pas certain qu'aucun corps existe, je sais, toutefois, que je n'ai pas ce qui appartiendrait à ces corps, de l'existence desquels je doute, et que je ne suis pas, particulièrement, en moi-même, ce corps auquel je suis attaché. Cela signifie que ce qui appartient aux corps ne m'appartient pas, et cela signifie que, lors même que je ne peux affirmer l'existence des corps, je vois que ce qui leur appartient ne m'appartient pas. Qu'est-ce qui m'appartient ? La pensée mathématique la plus simple m'informe que, soit que les corps existent ou non, ils sont de l'étendue géométrique. Par conséquent, quand je m'affirme comme chose qui pense, je me distingue - et cela en vertu de l'expérience même du doute que je viens de traverser - des corps qui sont de l'étendue. Ce qui signifie que, dans la certitude du Cogito, je pose, et que je suis une substance qui pense, et que je me distingue d'une substance étendue. Je suis une « res cogitans » et non une « res extensa ». Voilà donc, en vertu du Cogito, la saisie par le dedans, pour ainsi dire, de la notion de substance. Descartes ne peut, pour l'instant, dans le contexte premier du Cogito, abonder en explications sur la sub- 
stance dont il ne peut encore affirmer l'existence. Dans la Méditation $2^{e}$ et à la fin de la Méditation $5^{e}$, Descartes, quand il arrive à Dieu, se référera encore à l'expérience première du Cogito pour en tirer parti.

1.4 Avec cette réflexion cartésienne sur le Cogito, sur la proposition " je pense, je suis ", le point de départ d'une théorie de la substance est ainsi donné. En quel sens entendre le terme de substance dans l'affirmation " je suis une chose qui pense ", une substance qui pense ? En quoi l'expérience du Cogito éclaire-telle la notion qui nous concerne ici ? Tout d'abord, négativement parlant, la substance en question, dans le contexte que je viens de préciser, n'est nullement celle de la scolastique : il ne s'agit pas de la substance aristotélicienne, au sens d'un composé de matière et forme, puisqu'ici la matière est absolument exclue. En outre, on ne peut pas dire non plus que, pour Descartes, la substance est un sujet, un substrat comme le soutenait aussi

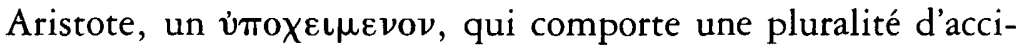
dents : tantôt une substance a telle qualité, tantôt telle autre, autant d'accidents. L'idée d'accident n'effleure nullement la pensée de Descartes : nous sommes dans le substantiel et nous ne sortons pas de là ! Faut-il entendre par substance (ce que dira en un sens Leibniz) le sujet d'une proposition logique, une sujet qui comporte des attributs au sens de prédicats ? Il n'est pas question de cela, non plus. Quand il s'agit de la substance, Descartes, au nom du Cogito, écarte toute explication logique.

Qu'est-ce donc que la substance? Le terme qui l'indique, en premier, se trouve dans la proposition célèbre : « je pense donc je suis " : je, moi, je suis une chose qui pense, je suis la chose qui pense. La substance est avant tout le je, le moi, c'està-dire ce qui est affirmé à partir du doute et par-delà le doute, ce qui tient devant le doute et devant toute espèce de danger ou de réalité qui pourrait vouloir me tromper. La substance c'est le moi et, puisque nous sortons du doute par l'affirmation même d'un je doute, d'un je pense, la substance c'est le moi libre, la liberté. Descartes en reviendra toujours là. Mais ce n'est encore ići qu'une conviction sous-jacente à toute la philosophie cartésienne, et la théorie de la substance ne s'en tient pas là. "Je suis une chose qui pense ". Il semble qu'il y ait comme une équivalence entre la chose et.le pensée. Mais comme la pensée 
ne peut être un accident de la chose, nous venons de le rappeler, ce serait donc son essence même ? Mais est-ce la même chose ? La substance serait-ce la pensée pour Descartes ? Ce serait inacceptable. Et Descartes abonde, aussi bien dans les Méditations que dans les Réponses aux Objections, et particulièrement dans les Réponses aux troisièmes objections, formulées par Hobbes, en explications pour nous montrer qu'il tient à sa phrase qui comporte une proposition relative : je suis une chose qui pense, moi qui sent, moi qui ${ }^{8}$; le sujet tient et se trouve clairement distingué de l'attribut. Sans doute, la pensée n'est pas un attribut quelconque, c'est l'attribut essentiel, et même l'attribut unique et toutes les autres activités de moi-même, comme de désirer, de douter, de sentir, ne sont, au fond, que des variations de cet attribut essentiel. Mais la forme même des expressions cartésiennes, chose qui, moi qui, etc., indique que la substance est, en elle-même, distincte de l'attribut, par-delà ou en-deça, comme on veut, de l'attribut. Dans les Entretiens de Descartes avec Burman, cela est tout à fait clair, malgré le ton familier et apparemment peu sérieux de Descartes. Aux questions de Burman, Descartes répond en précisant que la substance n'est pas l'attribut, ni plusieurs attributs, ni tout simplement la totalité des attributs (c'est ce que dira plus tard Spinoza). Descartes tient, comme Aristote, mais dans un sens tout à fait différent, à soutenir que la substance est la chose $1^{\text {ère }}$, l'objet de la philosophie $1^{\text {ère }}$, dans la mesure évidemment où cette substance est nousmêmes. Le rôle de cette pensée, chez Descartes, de la substance comme l'attribut essentiel, de l'attribut principal, et, pour ainsi dire, de l'attribut unique, a une importance extrême. Si je me connais comme substance dans la mesure où je pense, cela a pour conséquence que je ne connais pas directement, Descartes ne le relève pas explicitement, la substance, ou plus exactement c'est la pensée qui me révèle à moi-même, qui révèle cette substance que j'appelle moi-même. Cela signifie que chaque fois où une action m'est attribuée, je marche, je me nourris, je sens, en tout cela, je ne suis sûr que d'une chose, que moi qui pense, j'existe. Mais, de plus, chaque fois que j'affirme une proposition, que je juge, en somme, même si je dis : cette chambre est chaude,

8. Oeuvres de Descartes, éd. Adam-Tannery, IX, 135, 136-137, VII, 174-175-176. 
même si je dis le roi est mort, même si je dis, la somme des angles d'un triangle est égale à deux droits, l'affirmation : je pense donc je suis, est sous-jacente. Ma pensée me révèle et comme être et comme capable de connaissance. On peut même aller plus loin et soutenir que le « je suis une chose qui pense ", soutenir que la pensée est mon attribut essentiel, qu'elle est, dans un certain sens, mon essence même. Cela signifie, en somme, qu'en mon être il n'est, à proprement parler, autre chose que ne révèle, que ne montre clairement, que ne pose, ma pensée. Je suis, de part en part, pensée, (c'est-à-dire, comme dira plus tard Spinoza, conscience) et je ne suis que cela. L'être substance comme matériel est catégoriquement nié par Descartes. Il n'y a en moimême, et comme essentiel à moi-même, et, comme moi-même, que cette pensée, cette conscience.

1.5 Voilà le noyau de la théorie cartésienne de la substance telle qu'elle apparaît dans les Méditations et dans les Réponses aux objections lorsqu'on examine le rapport entre Cogito et Substance. Il faut néanmoins attendre les Principes pour avoir une conception technique de la substance. En effet, la $1^{\text {ère }}$ partie des Principes, à partir du paragraphe 48 , contient des développements importants sur la substance ; surtout c'est le paragraphe 51 qui donne la définition cartésienne de la substance. À cause de l'importance de cette définition et des problèmes qu'elle soulève, voyons-en tout de suite les termes et leurs conséquences. Descartes écrit ${ }^{9}$, pour commencer :

. . . Lorsque nous concevons la substance, nous concevons seulement une chose qui existe en telle façon qu'elle n'a besoin que de soi-même pour exister.

Descartes relève immédiatement cette expression car elle pourrait créer de l'ambiguïté et répond à l'objection possible en précisant qu'à proprement parler, cette expression " n'avoir besoin que de soi-même pour exister " s'applique exclusivement à Dieu. Dieu seul est la chose qui n'existe en telle façon qu'elle n'a besoin que de soi-même pour exister. Du reste, déjà dans les Réponses aux $1^{e ̀ r e s}$ Objections, quelque chóse de cela a été prévu. Dans la partie de ses réponses ${ }^{10}$ qui concernent Dieu, Descartes affirme

9. Oeuvres de Descartes, éd. Adam-Tannery, IX, II, 47.

10. Oeuvres de Descartes, éd. Adam-Tannery, IX, 87, VII, 109, 110. 
que Dieu est essentiellement la chose qui est par soi : " a se " et, plus loin, Descartes dit, en effet, que, dans un certain sens, Dieu est " cause de soi », bien que cette expression de " cause de soi " rappelle la cause efficiente et mécanique. Dieu est « causa sui ", comme dira plus tard Spinoza. Descartes dit bien cela : « j'avoue franchement qu'il peut y avoir quelque chose dans laquelle il puisse y avoir une puissance si grande et inépuisable qu'elle n'ait jamais eu besoin d'aucun secours pour exister et qu'elle n'en est pas encore besoin maintenant pour être conservée et ainsi qui soit en quelque façon la cause de soi-même et je conçois que Dieu est tel ${ }^{11} »$.

On comprend que Descartes se reprenne tout de suite quand il donne sa définition de la substance au paragraphe 51 des Principes ( $1^{\mathrm{ère}}$ Partie). Définir ainsi le terme de substance ne s'applique qu'à Dieu, et, de là, il n'y a apparemment qu'un pas pour dire, comme Spinoza, qu'il n'y a qu'une seule substance et que le reste, ce sont les attributs de cette substance unique, Dieu, que Descartes et Spinoza appellent du même terme : " causa sui ".

Mais Descartes se reprend, et tout de suite, en précisant en quel sens il peut y avoir de l'obscurité touchant l'explication de cette phrase " n'avoir besoin que de soi-même pour exister »; c'est pour cela qu'il ajoute ensuite, que l'École enseigne que le terme de substance n'est pas " univoque au regard de Dieu et des créatures, c'est-à-dire qu'il n'y a aucune signification de ce mot que nous concevions distinctement, laquelle convienne à lui et à elles ${ }^{12}$. Par conséquent, le terme, pour Descartes, pourrait s'employer pour autre chose que Dieu, et il admet explicitement que les créatures peuvent être dites des substances.

On pourra appeler substance ces créatures dans la mesure où il y a certaines choses créées qui ne peuvent exister sans quelques autres, par exemple la rondeur de la table par rapport à l'étendue, mon imagination par rapport à moi-même ou à ma pensée, toutes ces choses, nous devons les distinguer de celles

11. Ibidem.

12. Descartes, op. cit., A.T. IX, II, 47 ; titre du paragraphe 51 : ce que c'est que la substance ; et que c'est un nom qu'on ne peut attribuer à Dieu et aux créatures en même sens. 
qui, pour exister, n'ont besoin que du " concours ordinaire "; « du secours de Dieu pour exister ».

Y a-t-il là deux théories, deux acceptions, deux conceptions de la substance ? L'une qui conviendrait à Dieu, l'autre aux créatures, et particulièrement pour moi-même ? Descartes semble l'affirmer puisqu'il refuse l'univocité que proposera plus tard Spinoza. Mais il ne l'affirme pas complètement puisqu'il demeure quelque chose de commun, bien que ce quelque chose de commun ne soit pas très clair à dire, entre le "n'avoir besoin que de soi-même » et le « n'avoir besoin que du secours ordinaire de Dieu pour exister ». Ce qui serait commun serait, au fond, le terme d'exister mais Descartes est bien le philosophe le plus opposé à des termes communs qui ne signifieraient pour lui que des abstractions vides de sens. Parti du doute, il pose le moi en vertu d'une expérience privilégiée, d'une expérience qui est en même temps réflexion privilégiée, et petit à petit pose Dieu. En un certain sens, on ne peut parler d'un fondement commun de la théorie cartésienne de la substance et, en un certain sens, on est presque obligé de dire que Descartes accepterait que le terme de substance soit équivoque. La scolastique, on le sait, met entre l'univocité qui, pour la notion de substance, pourrait conduire au panthéisme, et l'équivocité qui conduit au refus de la pensée et, pour les philosophes, au nominalisme, entre l'univocité et l'équivocité, la scolastique pose, donc, l'analogie qu'on pourrait expliquer brièvement de la manière suivante, en ce qui concerne la substance : la substance par rapport à Dieu est comme la substance par rapport au moi, comme la substance par rapport au corps. Le fondement de l'analogie, pour la scolastique, est quelque chose de tout à fait net, et c'est ce qui nous permet de dire la substance par rapport à la doctrine de l'Être : l'homme est capable de connaître l'Être ; et en partant de l'Être, de l'objet même de l'ontologie, on pourrait faire une affirmation du type analogue ... Rien de tel chez Descartes : nous ne partons pas de l'Être, nous partons du doute, du je doute, du je pense . . .

Dans les Principes, l'approche du problème est, en définitive, toujours la même, encore que Descartes entre, sans doute, dans beaucoup de distinctions et qu'il consacre plusieurs paragraphes à la substance. Plutôt que de s'appuyer sur la définition de la substance au paragraphe 51, que Descartes accuse lui- 
même, ainsi qu'on vient de le voir, d'être équivoque, ${ }^{13}$, il faut recourir au paragraphe 60 , dans lequel il distingue les substances et parle de la « distinction réelle ${ }^{14}$, pour mettre en évidence ce que Descartes tient à dire sur la substance. Or ce qui est clair, d'après ce paragraphe, c'est que, pour Descartes, la substance étendue se distingue radicalement de la substance pensante. Le fondement de cette distinction est présenté ainsi : ... « parce qu'un chacun de nous apergoit en soi qu'il pense - c'est cela le fondement de la distinction - et qu'il peut en pensant exclure de soi ... toute autre substance ou qui pense ou qui est étendue $» \ldots{ }^{15}$. On est en présence de la même thèse, humaniste, volontariste, rencontrée dans les Méditations, bien que le langage des Principes soit plus explicite.

Et c'est bien sur ce point que le contraste entre les deux conceptions de la substance est le plus frappant. Ce qu'il y a de clair, chez Descartes, à propos de la substance, c'est ce qu'il dit à propos du moi. On dirait que la notion perd de sa clarté quand elle sort de ce domaine. Qu'est-ce au fond que la substance pensante ? Ce serait assez peu dire que de dire, " c'est une réalité qui a l'attribut de la pensée "; d'un tour par trop scolastique au gré de Descartes. L'attribut est identifié à la substance en ce sens que la pensée ou la conscience révèle la substance, me révèle à moi-même et comme être et comme capable de connaissance ; telle est la signification de l'enchainement du je doute, je pense, je suis. C'est pourquoi Descartes pourra dire, plus tard, dans les Principes, paragraphe $60:$ " parce qu'un chacun de nous ... et qu'il peut en pensant exclure de soi tout autre substance ou qui pense . . . ${ }^{16}$. Quand Descartes essaie de comprendre la substance, il la comprend, comme l'indique déjà le paragraphe 51, ambiguë et équivoque, comme une réalité indépendante, une réalité qui a l'indépendance et qui en jouit ; de là la conception de la substance comme "une chose qui existe en telle façon qu'elle n'a besoin que de soi-même pour exister »; de là, la raison de la correction de Descartes, dans le même paragraphe : la substance est ce qui n'a besoin pour exister que « du concours

13. Ibidem.

14. Descartes, op. cit., \#60 ; A.T. IX, II, 51.

15. Descartes, ibidem.

16. Descartes, ibrdem. 
ordinaire de Dieu ${ }^{17}$. En disant cela Decartes pense au moi et il déclare nettement, dans le paragraphe 60 , qu'on peut exclure de soi toute autre substance qui pense. Ce qui signifie que cette aperception en soi, qui n'est rien d'autre que le cogito, lui révèle non seulement son existence, mais aussi sa distinction d'avec ce qu'il ne pense pas, et surtout d'avec d'autres substances qui pensent, qui peuvent penser et exister à part lui, c'est-à-dire sa distinction des autres, et avant tout de Dieu. La Première Méditation sur le doute avait d'ailleurs préparé à cette saisie du moi comme distinct d'autres esprits : dans cette $1^{\text {ère }}$ Méditation, il s'agit de Dieu et du Malin Génie, dans les suivantes, des autres qui peuvent penser comme moi mais qui ne sont pas moi. En résumé, la substance chez Descartes est avant tout saisie comme personne.

1.6 En est-il de même pour Leibniz qui reprend en la développant la tradition aristotélicienne et scolastique concernant la substance et les formes substantielles ? En ceci il tient à être plus conforme que Descartes à la tradition et à sa terminologie, ce qu'on ne peut pas dire pour Descartes pour qui la substance c'est le Je Pense. L'approche de Leibniz est cependant avant tout logique, comme je le disais tout à l'heure (proposition - sujet - prédicat ; prodicatum inest subjecto), logico-grammaticale, comme dirait $\mathrm{Ch}$. Serrus. On verra qu'elle est de plus, ontologique, cosmologique - Leibniz rapproche la substance et le monde - et aussi théologique.

Dans son étude de 1694, De prima philosophice emendatione, Leibniz considère, ceci en liaison avec ses travaux du moment en physique, qu'il faut partir de la définition scolastique de la substance comme vis activa ${ }^{18}$, comme l'être qui a en soi la source de son activité, pour établir en métaphysique une définition moderne et « véritable » de la substance. Il écrit : «Pour donner quelque indication de cette chose, je dirais que la notion des forces . . . à l'explication de laquelle j'ai consacré la science particulière de la Dynamique, apporte beaucoup de lumière pour

17. Descartes, op. cit., \#51, A.T. IX, II, 47.

18. Je rappelle que les deux autres définitions de la substance dans la scolastique sont celles de la substance a) comme le dernier support de toutes les propriétés et de toutes les déterminations (ultimum subsistens), b) comme ce qui demeure immuable sous tous les changements (ultımum perdurabile). 
comprendre la vraie notion de substance. La force active se distingue en effet de la puissance nue que connaissent les écoles en ce que la puissance active des scolastiques, ou faculté, n'est rien d'autre que la possibilité proche d'agir qui a cependant besoin de recevoir, pour passer à l'acte, l'excitation, et pour ainsi dire l'aiguillon d'une autre chose. Mais la force active contient un certain acte ou $\varepsilon \dot{\nu} \tau \varepsilon \lambda \varepsilon_{\chi} \varepsilon\llcorner\alpha \nu$, elle est intermédiaire entre la faculté d'agir et l'action elle-même, et elle comprend un effort ; et ainsi elle se porte d'elle-même à l'opération sans avoir besoin d'aides mais par le seul fait que l'obstacle est écarté ${ }^{19}$.

L'idée importante ici, et qui constituera un des points de fixation de la théorie leibnizienne de la substance, est que la substance est un être capable d'action. Leibniz s'accorde en cela avec la définition de l'être comme $\delta u ́ v \alpha \mu$ เs dans le Sophiste, avec

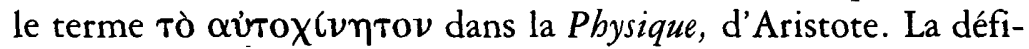
nition de la substance résumée à un être capable d'activité se retrouvera dans les Principes de la nature et de la grâce fondés en raison (paragraphe I, E 714a), dans la Théodicée (paragraphe 400, E 618 b, GP.VI, 354) où Leibniz ajoute le terme de "spontanéité ", ainsi que dans la Réponse aux Réflexions, parue en 1709 dans le Journal des Sçavants (Rep. E 960 a ; GPIV, 594) : "Ce qui n'agit pas ne mérite pas le nom de substance ".

1.7 Avec cette première ligne de définitions de la substance, comme ce qui possède en sa propre spontanéité le principe de son agir et de son pâtir, et que l'on doit mettre en relation avec les conceptions leibniziennes dynamiques et mathématiques, liées au calcul infinitésimal (à partir de 1677-78) et du rejet du mécanisme cartésien, on peut repérer une seconde ligne de définitions qui insistent sur l'individu visant individuel comme constituant, seul, l'ens reale, l'unique réalité, et partant, en ce qui concerne la notion substance, son " principe fondamental ", comme dira Hegel dans son Histoire de la philosophie, "est l'individuel " (Leçons sur l'bistoire de la philosophie, XIX, 449). Le paragraphe 63 de la Monadologie (1714) version finale du système énonce : « le corps appartenant à une monade qui en est l'entéléchie ou âme constitue avec l'entéléchie ce qu'on peut appeler un vivant,

19. Leibniz : De prima philosopha emendatione, et de natione substantia, E $122 \mathrm{a} / \mathrm{b}$, Gerhardt, IV, 469, Berlin 1875. 
et avec l'Âme, ce qu'on appelle un animal (M. paragraphe 63, E 701 a, GPVI, 617).

1.8 Revenons au Discours de Métaphysique, en particulier au paragraphe VIII, et à la manière dont Leibniz définit la substance. Cette définition a l'avantage de la rigueur ; de plus elle est faite en rapport avec les études logiques de Leibniz, qui chez lui ont commencé de très bonne heure et dont les notions vont interférer, en ce qui concerne la notion de la substance, non seulement avec toutes les étapes de sa doctrine mais avec aussi bien ses conceptions mathématico-physiques. Le Discours de Métaphysique, fin 1685, l'un des plus beaux textes de Leibniz, ainsi que le note Y. Belaval, a surtout l'avantage de mettre en place tous les thèmes essentiels concernant la substance, bien que la prodigieuse activité de Leibniz est loin de toucher à son terme, et que la correspondance avec le $P$. des Bosses servira à élaborer la doctrine de la substance composée. Mais on peut dire avec Belaval, qu'en 1686 le système est précisé et «qu'il ne manque guère au $\mathrm{D}$. de Métaphysique pour être la Monadologie que le nom même de " monade " employé seulement à partir de 1695. Que définit-il en fait dans le paragraphe VIII ? Non seulement annonce-t-il, dans ce paragraphe, qu'il définira la substance mais il dit clairement qu'il expliquera " en quoi consiste la notion d'une substance individuelle ${ }^{20}$. Le problème est donc ici de définir la substance individuelle - un des plus importants motifs, signalerais-je au passage, qui ont poussé Leibniz à se distinguer de Spinoza et à écrire le Discours de Métaphysique. Alors que Descartes est certain de la substance à partir du cogito, de l'aperception en soi, ainsi de suite . . . Leibniz élabore sa théorie de la substance à partir de nombreuses études et dynamiques et mathématiques $^{21}$, à partir du principe des indiscernables, et, en somme à partir d'une expérience des individus. Ce n'est pas en soi-même que Leibniz regarde. En habitué de la diplomatie

20. Leibniz, op. cit., D.M. p. 35 \#XIII.

21. L'idée mathématique d'infini, et surtout du changement infinitésimal d'une part, d'une loi pour la production, pour ainsi dire, des infiniment petits, d'autre part, sont d'une importance considérable, comme l'ont montré ses commentateurs, pour la genèse de la métaphysique leibnizienne. De même, l'idée de force et de la constance de la force que Leibniz introduit dans la mécanique, toute sa critique de la mécanistique carétisienne, aurait alimenté sa réflexion sur les notions métaphysiques fondamentales. Cf. sur ce point, notamment, l'étude de J. Jalabert : La théorie leibnizienne de la substance. P.U.F. 1947. 
et des petites cours d'Allemagne, il regarde les autres et distingue entre individus.

1.9 La définition de la substance, donnée au paragraphe VIII du Discours a donc l'avantage de la rigueur. Mais il n'y a pas que cela ; autrement dit, la substance, chez Leibniz, n'est pas seulement conçue comme le sujet d'une proposition identique ou analytique. La définition logique qui est donnée au paragraphe VIII est l'expression de réflexions extra ou infra logiques, de réflexions métaphysiques : au paragraphe XII, lorsque Leibniz soutient contre Descartes que l'étendue n'est pas une substance puisque, selon la définition donnée au paragraphe VIII, la substance est une réalité qui a de l'unité, il indique aussi que la substance doit être de l'ordre spirituel, de l'ordre de l'âme : " . . . mais qu'il faut nécessairement y reconnaître quelque chose qui aye du rapport aux âmes, et qu'on appelle communément forme substantielle ... . ${ }^{22}$. C'est pourquoi Leibniz parlera plus tard de la monade pour dire la même chose que la substance individuelle, laquelle est, dans le langage néo-platonicien repris par G. Bruno, l'unité spirituelle. En plus de l'origine logique du langage leibnizien sur la substance, on peut par conséquent montrer, à même le Discours de Métaphysique, qu'il y a une origine dynamique, mécanique. Dès les premières lignes du paragraphe VIII, Leibniz dit " qu'il y en a qui croient que Dieu fait tout, d'autres s'imaginent qu'il ne fait que conserver la force qu'il a donnée aux créatures - or puisque les actions et passions appartiennent proprement aux substances individuelles, il serait nécessaire d'expliquer ce que c'est qu'une telle substance ». Ces trois mots, force, action et passion, appartiennent au langage de la dynamique. À l'encontre de Descartes, les conceptions de Leibniz en mécanique lui font affirmer une force, la réalité de la force. Il remonte, au-delà de l'étendue, au mouvement, et du mouvement à la force. Et l'étude proprement mécanique du mouvement l'invite, en dehors de toute expérience psychologique, à affirmer des unités de force dans le monde physique. Et évidemment, en physique, il ne peut pas aller plus loin. D'ailleurs l'expérience ne nous donne pas ces unités de force, les mathématiques, non plus. Nous sommes invités à les cher-

22. Leibniz, op. cit., D.M. p. 41, \#XII. 
cher en dehors de la physique, en pleine métaphysique. C'est sur ce terrain que nous rencontrons la substance et, comme on le verra, en vertu de la préparation dynamique et mécanique, plusieurs substances.

1.10 Le langage du Discours de Métaphysique est aussi, en même temps, un langage mathématique : Leibniz y parle courbe, équation ; un langage de mathématicien opticien : miroir, perspective. Et sa mathématique n'est pas celle de Descartes qui part d'une notion et qui la développe, Leibniz, lui, part de plusieurs notions ou de plusieurs problèmes et sa recherche privilégiée, a-t-on dit, c'est de trouver plusieurs types d'équations, différentes entre elles et qui rendraient compte de la variété de chaque réalité. Le trait d'union qui permet à Leibniz de passer de cette variété de courbes ou d'équations à une variété de substances est la notion de l'infini par laquelle il se trouve en avance sur Descartes. Ses études sur le calcul infinitésimal ne sont certes pas étrangères à ses réflexions sur la substance ${ }^{23}$ mais peut-on dire que ses réflexions mathématiques sont la source de sa théorie de la substance?

$1.11 \mathrm{Je}$ pense qu'aussi bien les réflexions en physique qu'en mathématiques sont postérieures à une réflexion essentielle, aussi essentielle chez Leibniz que l'expérience du doute l'est chez Descartes, elle qui le conduit à la découverte du moi et au rattachement au monde et à la pluralité des substances pensantes. En face de cette réflexion cartésienne essentielle, il faut trouver celle essentielle leibnizienne. Le centre de la pensée de Descartes pour la substance c'est le Je Doute, le Je Pense, le Je Suis. Quel est le centre pour Leibniz ? Il se place du point de vue du monde, de quelqu'un qui regarde le monde, à l'inverse de l'attitude cartésienne. Malgré son idéalisme, Leibniz se place au point de vue du monde, et en regardant le monde, il voit non seulement la pluralité des substances mais surtout il est obligé d'en tenir compte. Plus fortement encore, je dirais qu'en se plaçant ainsi de ce point de vue du monde, Leibniz essaie de trouver la théorie de la substance en fonction de ce point de vue cosmologique -

23. C'est Bréhier, je crois, qui disait, je cite de mémoire, que toute la philosophie de Leibniz est en chaque question la découverte d'une sorte d'algorithme qui joue, mutatis mutandis, le rôle de l'algorithme infinitésimal dans le calcul de l'infini. 
alors que pour Descartes le point de vue est psychologique plutôt, il se place au point de vue de Dieu qui regarde le monde, au point de vue théologique. Ce n'est plus moi qui regarde le monde, c'est Dieu, et Dieu regardant le monde et le tournant de chaque côté, c'est la substance (cf. le paragraphe 14 du Discours de Métaphysique). Leibniz, en somme, pour définir la substance, se place au point de vue de l'idéalisme théologique et Descartes au point de vue de l'idéalisme psychologique, pratique.

2. LA SUBSTANCE ET SON CONTENU.

Après avoir envisagé la manière dont l'un et l'autre philosophe définissent la substance, et comment ils la conçoivent - une personne pour Descartes, un individu-sujet pour Leibniz — il faut maintenant préciser son contenu.

2.1 Pour déterminer quel est le contenu de la substance pour Descartes, on n'est pas obligé de s'arrêter, comme tout à l'heure, au psychologisme premier ou à l'humanisme premier ou encore au volontarisme premier. C'est à propos du contenu de la substance que Descartes développe, ou en tous les cas indique, sa théorie des attributs. Implicite dans les Méditations, celle-ci est, sans beaucoup de changements, explicite dans les Principes. L'âme est une substance, elle a pour attribut principal, ou essentiel, la pensée : c'est pourquoi elle est appelée res cogitans : cette expression indique, par elle-même, que la pensée est davantage qu'attribut principal ou essentiel, elle est attribut unique. De même pour le corps : une substance étendue dont l'étendue est attribut principal mais en fait attribut unique (paragraphe 53 des Principes, I). Ce serait peut-être là une des sources d'inspiration de la théorie moniste de la substance chez Spinoza. Mais que signifie cet attribut principal ou unique de la substance, quel est son rôle? S'il est identique à la substance pourquoi l'évoquer ? Descartes parlant ensuite de volonté, de pensée, de doute, les appellera des propriétés de l'âme ou modalités. Comment comprendre donc le sens de l'attribut ? Est-ce simplement ce qui qualifie la chose (pensante), l'âme, comme, par exemple, la trompe qualifie avant tout l'éléphant ou ses défenses ? Pas du tout. L'étendue ne qualifie pas, ce serait encore de la scolastique. L'attribut de la pensée, c'est la conscience, c'est ce qui révèle 
l'homme à lui-même. Chaque fois que je dis que je pense ou chaque fois que jagis comme moi-même, je suis conscient de ce que je fais, je sais et je m'affirme comme existant, comme voulant. Ceci est clair pour l'âme. Quel est le rôle de l'étendue ? Il s'agit de quelque chose d'analogue, de parallèle : l'étendue non plus ne qualifie pas le corps, l'étendue révèle le corps. Elle est, si l'on veut, le corps lui-même, pas pour le corps puisque celui-ci ne se pense pas, mais elle le révèle à moi-même.

2.2 Il n'est pas question de tout cela chez Leibniz. Quand celuici parle d'attribut, il entend d'ailleurs tout à fait autre chose. L'attribut, pour Leibniz, est le prédicat. Sa conception de l'attribut est une conception logique et grammaticale. L'attribut c'est ou le prédicat ou la qualité, les accidents, non pas l'essence, comme chez Descartes. L'insistance, chez Leibniz, porte sur la substance-sujet. Alors que l'attribut est pris très au sérieux par Descartes lorsqu'il s'agit de l'âme et même lorsqu'il s'agit du corps, il ne l'est pas par Leibniz, ou plutôt l'attribut n'est pas, métaphysiquement ou ontologiquement parlant, considéré sérieusement par Leibniz quand il s'agit du plan de la créature ; il ne l'est que lorsqu'il s'agit du plan de Dieu : l'attribut entendement, l'attribut volonté. Pour la créature, Leibniz parlera de qualités, pour l'âme humaine, de fonctions. La fonction de l'âme " a cependant quelque chose d'essentiel ", et d'essentiel à la substance créée. Et quelle serait alors, selon Leibniz, cette fonction " essentielle? "Il ne faudrait pas dire tout de suite que c'est la perception, l'appétion ou la tendance ; ce ne sont là après tout que des notions subordonnées. La fonction essentielle et première de la substance créée, celle qui se dégage de la constitution même de la substance, est l'expression ; c'est ensuite la représentation; c'est enfin la perception. Quant à la tendance qui caractérise le passage d'une perception à une autre, elle caractérise avant tout l'indépendance à la substance, le fait de son action, de son activité.

2.3 Alors que pour. Descartes ce qui est essentiel pour l'âme c'est la pensée, ou encore davantage la conscience, le pour-soi, comme diraient les contemporains, pour Leibniz l'essentiel de la substance est d'exprimer, ensuite de représenter ensuite de percevoir. Ce n'est que dans quelques substances, ou comme le dit la Monadologie, dans quelques monades, que la perception 
devient l'aperception, c'est-à-dire la pensée, la conscience. Si pour Descartes, paradoxalement, l'âme pense toujours, pour Leibniz elle ne pense pas toujours, elle perçoit toujours, ou plutôt elle représente, elle exprime toujours. La conception leibnizienne de la substance, par cette notion même d'expression, n'est pas seulement, par conséquent, une conception logicogrammaticale mais une conception cosmologique. La notion d'expression ressortit à une notion cosmologique. Elle se dégage, chez Leibniz, quand celui-ci ayant à dire ce qu'est la substance individuelle tient à dire en même temps ce qu'elle est dans le monde, par rapport au monde : si Dieu la crée, il l'a créée comme miroir de l'univers ${ }^{24}$. Cette conception de la monade, miroir de l'univers, enveloppe également une raison théologique : Dieu crée pour sa gloire ; chaque substance est faite pour magnifier sa puissance, sa sagesse et sa bonté. La variété glorifie sa puissance ; l'ordre, sa sagesse, et la perfection c'est-à-dire la variété dans l'ordre, exprime sa bonté $e^{25}$. Chaque substance est miroir de l'univers mais aussi miroir de la Divinité : « . . . toute substance est comme un monde entier et comme un miroir de Dieu ou bien de tout l'univers, qu'elle exprime chacune à sa façon, à peu près comme une même ville est diversement représentée selon les différentes situations de celui qui la regarde. Ainsi l'univers est en quelque façon multiplié autant de fois qu'il y a de substances, et la gloire de Dieu est redoublée de même par autant de représentations toutes différentes de son ouvrage. On peut même dire que toute substance porte en quelque façon le caractère de la sagesse infinie et de la toute puissance de Dieu, et l'imite autant qu'elle en est susceptible ${ }^{26}$.

24. Leibniz, La Monadologie, éd. par Boutroux chez Delagrave, Paris, 1881 ; paragraphe 56 : " chaque substance simple est un miroir vivant perpétuel de l'univers "; paragraphe 57 : « il y a comme autant de différents univers qui ne sont pourtant que les perspectives d'un seul selon les différents points de vue de chaque Monade ».

Ch. Rodier in Revue de Métaphysique et de Morale, sept. 1902, p. 338, fait remarquer dans son article "Sur une des origines de la philosophie de Leibniz ", les emprunts de Leibniz aux métaphores plotiniciennes et comment Leibniz a signalé lui-même, la parenté de sa conception de la monade, miroir de l'univers, avec la thèse plotinienne de l'âme intelligible (in Dutens, Opera Omnia, II, 1, p. 223).

25. Cf. Leibniz, Monadologie, op. cit., paragraphes 57-58-59.

26. Letbniz, Discours de Métaphysique, Op. cit. paragraphe IX, p. 37. 
3. RAPPORT DES SUBSTANCES.

La conception leibnizienne de la substance est donc essentiellement une conception logique et cosmologique qui pose l'expression comme le contenu essentiel de la substance alors que la conception cartésienne est apparue radicalement psychologique, volontariste : la substance pensante c'est la conscience. La considération et la comparaison des théories cartésienne et leibnizienne au sujet des rapports des substances entre elles, feront apparaître enfin une dernière différence entre le substantialisme de chacun des philosophes. Le problème du rapport des substances présuppose cependant lui-même qu'on prenne position relativement à la question de savoir s'il y a une ou plusieurs substances, chez l'un ou chez l'autre, et quel est le sens de l'unicité, s'il y a unicité, le sens de la pluralité, s'il y a pluralité ; on pourra, ensuite, parler du rapport entre les substances.

3.1 Sur la question de l'unicité ou de la pluralité des substances, on sait que l'un comme l'autre philosophe évite l'unicité que Spinoza a réaffirmée. Pour Descartes, on parlera surtout de pluralité. Il faut soutenir, pour bien comprendre la position de ce dernier, qu'il existe, chez lui, deux sens de la pluralité, qu'il est pluraliste de deux manières. Il y a d'abord une pluralité de types de substances, et, avant tout, il y a dualité de la substance. Mais comme la différence est infinie entre la substance créée et la substance créatrice, on ne peut dire que la substance s'entend en ce qui concerne Dieu de ce qu'elle s'entend en ce qui concerne l'âme ou le corps. Si l'on s'en tient aux Principes, on peut parler ainsi de deux types de substances, de trois au maximum, mais si l'on se rapporte aux Lettres à la princesse Élizabeth, surtout à celles du 21 mai 1643 et du 28 juin $1643^{27}$, on est invité à considérer un autre type de substance : l'union de l'âme et du corps. Descartes parle à ce sujet d'une «notion primitive », et, par conséquent, irréductible à celle de l'âme ou à celle du corps. Lorsqu'on envisage cette union, on envisage, dit-il, une chose, c'est-à-dire si on reprend le langage de la Seconde Méditation, une substance. C'est pourquoi Descartes peut déclarer, au sujet de l'union de l'âme et du corps, que l'âme est étendue, tout en précisant bien qu'il s'agit d'une étendue distincte de l'étendue purement matérielle, et en entendant par là, par l'union de l'âme

27. Descartes, AT. III, 663 et sqq. ; 690 et sqq. 
et du corps, que l'âme et le corps dans l'union sont comme une et même chose, une même substance ${ }^{28}$. On voit qu'on a affaire à une pluralité de types. Un autre sens de la pluralité chez Descartes, et auquel j'ai déjà fait allusion, tient à la pluralité de la substance personnelle : je me réfere ici au paragraphe 60 des Principes, I.

3.2 En ce qui concerne Leibniz, la question de savoir s'il y a une ou plusieurs substances, le sens de l'unicité, s'il y a unicité, de la pluralité, s'il y a pluralité, se pose autrement. Alors que chez Descartes on a nettement affaire à une pluralité de types, chez Leibniz, c'est à une unicité de types. Toutes les monades, ou toutes les substances individuelles - l'appellation est indifférente chez lui - ont quelque chose d'essentiel, qui est partout identique, à savoir, l'expression, la représentation, la perception, partout que l'on envisage l'homme ou les êtres inférieurs à l'homme, peut-être même, pour Leibniz, jusqu'au minéral. Il y a dans le minéral non seulement une âme de vérité mais tout simplement une âme, dans la mesure où le minéral se réduit à des réalités substantielles, à des monades. Il n'y a pas, cependant, qu'unicité chez Leibniz, il y a aussi pluralité. Si, chez Descartes, la pluralité est une pluralité de personnes, il s'agit, chez Leibniz, d'une pluralité d'individus, et même d'une infinité d'individus ; c'est par là que Leibniz croit pouvoir se distinguer et se défendre de tout panthéisme. Dira-t-on qu'en vertu de l'unicité Leibniz penche vers un monisme d'allure spinoziste ? Il y a bien un type de substance, mais différents degrés, peutêtre même une infinité de degrés comme il y a une infinité d'individus.

3.3 En ce qui concerne maintenant le rapport entre les substances, il est très difficile à comprendre chez Descartes. Ce dernier ne part pas du monde ; il part de lui-même et arrive à Dieu ; c'est à partir de là qu'il essaie de redescendre et de poser un monde, et de concevoir, tant bien que mal, les rapports entre les substances. Toutes ses difficultés au sujet de l'union de l'âme et du corps en dérivent, ainsi que des difficultés qui ne sont même pas mentionnées concernant le rapport de moi-même avec les autres moi. Chez Leibniz, le problème du rapport des sub-

28. Cf. Descartes, Lettres à Élizabeth, du 28 juin 1643 ; A.T. III, 692. 
stances est évidemment résolu en principe dès le début. Leibniz ne pense pas, en effet, en fonction de lui-même mais il pense la substance en fonction du monde et de Dieu. Il se place au cœur du monde, et pour ainsi dire, dans la peau de Dieu.

Ni Descartes ni Leibniz ne reviennent à la théorie aristotélicienne de la substance : comme la matière première, indéterminée, ou de la substance, comme forme de l'être, ou de l'essence, définissable par une définition essentielle, ni à la substance, être composé de' forme et de matière. Tout cela serait verbeux pour un Descartes qui commence avec un cogito et l'expérience du doute, et pour un Leibniz qui commence avec la logique et avec le monde, et avec la mécanique et l'infini mathématique, et aussi avec un Dieu. Descartes s'achemine vers une théorie de la substance qui donne la priorité au moi et, par conséquent vers un idéalisme psychologique. Peut-on parler d'un réalisme de la substance chez Leibniz? Pas vraiment, car le moment décisif, à propos de la substance, est le moment où Leibniz essaie de remonter à la pensée divine, à Dieu, c'est-àdire à Celui qui pense l'univers et qui le regarde de tous les côtés, et où il affirme que le résultat de chaque regard de Dieu est une substance ${ }^{29}$ :

Car Dieu tournant, pour ainsi dire, de tous côtés et de toutes les façons, le système général des phénomènes qu'il trouve bon de produire pour manifester sa gloire, et regardant toutes les faces du monde de toutes les manières possibles, puisqu'il n'y a point de rapport qui échappe à son omniscience, le résultat de chaque vue de l'univers, comme regardé d'un certain endroit, est une substance qui exprime l'univers conformément à cette vue.

c'est ce que j'appellerai un idéalisme théologique à partir duquel Leibniz retrouve la théorie néo-platonicienne de l'expression et les fonctions de la substance.

Département de philosophie

Université du Québec à Montréal

29. Leibniz, Discours de Métaphysique, op. cit. p. 47, paragraphe XIV . 